\section{P4.031 DIFFERENCES IN CONDOM USE CONSISTENCY DURING VAGINAL AND HETEROSEXUAL ANAL SEX: FINDINGS FROM THE SAFE IN THE CITY STUDY}

\author{
doi:10.1136/sextrans-2013-051184.0929
}

'L H D'Anna, ' 0 A Korosteleva, 'D Perez, ${ }^{2} \mathrm{~L}$ O'Donnell, ${ }^{3} \mathrm{C}$ A Rietmeijer, ${ }^{4} \mathrm{~J}$ D Klausner, ${ }^{1} \mathrm{C}$ K Malotte, Safe in the City Study Group. 'California State University, Long Beach, Long Beach, CA, United States; ${ }^{2}$ Education Development Center, Boston, MA, United States; ${ }^{3}$ Denver Public Health, Denver, CO, United States; ${ }^{4}$ University of California, Los Angeles, Los Angeles, CA, United States

Background Condom use consistency is influenced by factors including participant and partnership characteristics, sex acts and cognitive mediators. We aimed to: (1) assess condom use consistency among urban U.S. STD clinic users; (2) examine condom use patterns for anal and vaginal sex within partnerships; and (3) identify characteristics and cognitive factors associated with condom use consistency.

Methods Patients $(n=1609)$ enrolled in a behavioural sub study of Safe in the City, a video-based STD/HIV prevention intervention, completed baseline and 3-month surveys measuring prior 3-month behaviours. The study population was restricted to 360 heterosexual males and females who reported both anal and vaginal sex with their most recent partner. Partnerships were classified into five mutually exclusive groups (below) according to reported condom use behaviour. Chi-square and Kruskal-Wallis tests assessed associations between condom use consistency and categorical (e.g., race, partnership type) and continuous (e.g., number of anal and vaginal sex acts, condom use beliefs) variables.

Results Of 360 partnerships, 156(43.3\%) reported no condom use; $92(25.6 \%)$ used condoms for vaginal but not anal sex; $9(2.5 \%)$ used condoms for anal but not vaginal sex; $61(16.9 \%)$ used condoms for anal and vaginal sex inconsistently $(<100 \%)$; and $42(11.7 \%)$ used condoms consistently (100\%) for both. Main partnership status was associated with increased inconsistent condom use in anal and vaginal sex. Consistent condom use for anal and vaginal sex was associated with the following cognitive mediators: anticipated partner agreement to future condom use, high condom self-efficacy with that partner, and positive condom-use beliefs.

Conclusions Condoms were not used over $40 \%$ of the time, and only a small fraction of partnerships used condoms consistently for both anal and vaginal sex. Results suggest that condom use counselling should consider barriers and facilitators unique to anal and vaginal sex, and that improving cognitive mediators may enhance the success of risk-reduction strategies.

\section{P4.032 YOUNGER GAY AND BISEXUAL MEN'S CONDOM USE WITH MAIN SEXUAL PARTNER IN NEW ZEALAND}

doi:10.1136/sextrans-2013-051184.0930

'N J Lachowsky, ${ }^{2} \mathrm{P}$ J W Saxton, ${ }^{3} \mathrm{~A}$ J Hughes, ${ }^{2} \mathrm{~N}$ P Dickson, ${ }^{1} \mathrm{~A} J \mathrm{~S}$ Summerlee, ${ }^{1} \mathrm{C} E$ Dewey. 'University of Guelph, Guelph, ON, Canada; ${ }^{2}$ University of Otago, Dunedin, New Zealand; ${ }^{3}$ New Zealand AIDS Foundation, Auckland, New Zealand

Background Main sexual partners are an important source of new HIV infections among MSM. Additionally, receptive (compared with insertive) anal intercourse carries considerably greater risk of HIV transmission. We investigated younger MSM's (YMSM, aged 16-29) condom use in New Zealand by main partner type and anal modality.

Methods The study included 1,221 YMSM who reported anal intercourse with a main partner (boyfriend or fuckbuddy) in the last six months from 2006, 2008 and 2011 rounds of the Gay Auckland Periodic Sex Survey and Gay men's Online Sex Survey. Condom use for both receptive and insertive anal intercourse was categorised into three levels: high (always/almost always), medium (about half the time), or low (very rarely/never). Associated factors were identified with four backward stepwise multivariate multinomial logistic regression models retaining at $p<0.05$.

Results The majority of YMSM classified their main regular partner as a boyfriend (59.5\%). Overall, high condom use (receptive and insertive) was more likely with a fuckbuddy $(59.2 \%$ and $59.4 \%$ ) than a boyfriend ( $35.8 \%$ and $37.5 \%$ ). Condom use was lower among YMSM in a relationship longer than one year, and was more common among those who used them more with casual partners. YMSM who only had insertive anal intercourse with their boyfriend were more likely to report high condom use compared with YMSM who also had receptive anal intercourse. However, compared with YMSM who were both insertive and receptive with casual partners, YMSM who were exclusively insertive with casual partners were less likely to report high condom use during insertive anal intercourse their boyfriend. YMSM who believed condoms reduce sensitivity were less likely to report using condoms.

Conclusion Condoms remain the leading STI primary prevention tool for sexually active YMSM. Sexual health education to improve condom use and discussions about extra-relational sex should be provided with consideration to anal modality.

\section{P4.033 EXAMINING DEPRESSIVE SYMPTOMS AND SUBSTANCE USE IN HIGH RISK MEXICAN AMERICAN AND AFRICAN AMERICAN ADOLESCENT WOMEN}

doi:10.1136/sextrans-2013-051184.0931

C C Young, J D Champion. The University of Texas at Austin, School of Nursing, Austin, TX, United States

Background Evaluate depressive symptoms and substance use among high risk Mexican (MA) and African (AA)-American adolescent women and identify factors associated with substance use to inform development of community-based interventions for high risk adolescents.

Methods AA ( $\mathrm{n}=94,16.8 \%)$ and $\mathrm{MA}(\mathrm{n}=465,83.2 \%)$ women (14-18 years) with histories of sexually transmitted infection (STI) or violence and high risk sexual behaviour were recruited from metropolitan public-health clinics. Participants completed semistructured interviews including assessments for violence, sexual risk behaviour, personal, family, peer, and partner substance use, depressive symptoms, and psychological stressors.

Results Most participants (82\%) reported elevated depressive symptoms (Centers for Epidemiologic Studies Depression Scale > 16) which were significantly associated with history of STI $(p=0.049)$. MA adolescents were more likely than AA adolescents to report high levels of psychological stressors $(t=-2.38, p=0.018)$, cigarette $(74 \%$ vs. $52 \%$, p < 0.001$)$, alcohol $(79 \%$ vs. $60 \%$, p < 0.001$)$, and other drug use $(87 \%$ vs. $67 \%, p<0.001)$. Substance use was associated with history of STI for MA adolescents only $(p=0.047)$. Over $79 \%$ of participants reported having a partner who used substances. No differences in STI history, depressive symptoms, physical, sexual or emotional violence, or partner substance use were identified by ethnicity. Controlling for theoretically guided variables (i.e., ethnicity, history of violence or STI, sex partners, life stressors, pregnancy), multinomial logistic regression analysis indicated ethnicity, numbers of sexual partner and depressive symptoms were predictors of substance use $(\mathrm{X} 2(6, \mathrm{~N}=554)=53.22$, $\mathrm{p}<0.001)$. Participants with substance using partner were more than two times as likely to use substances themselves.

Conclusions Findings suggest interventions aimed at reducing the incidence of STI should include comprehensive protocols that address depressive symptoms and substance use, particularly with $\mathrm{MA}$ adolescents. The role of partner substance use is also a potential target for intervention. 